\title{
Tragbare und selektive Gassensoren
}

\author{
Andreas T. Güntner ${ }^{1}$ \\ ${ }^{1}$ Klinik für Endokrinologie, Diabetologie und Klinische Ernährung, \\ UniversitätsSpital Zürich und Universität Zürich, Zürich, Schweiz \\ Kontakt: andreasthomas.guentner@usz.ch
}

\section{Einleitung}

Tragbare und kostengünstige Gassensoren bieten vielversprechende Möglichkeiten zur Realisierung kompakter Geräte für die flächendeckenden Überwachung der Luftqualität ${ }^{[1]}$, der Produktion von Lebensmitteln ${ }^{[2]}$, für die schmerzfreie, medizinische Atemluftdiagnostik ${ }^{[3]}$ (Abb. 1) und für die Suche und Rettung von Menschen ${ }^{[4]}$, um nur einige der unmittelbaren Anwendungen zu nennen.

\section{Air quality}

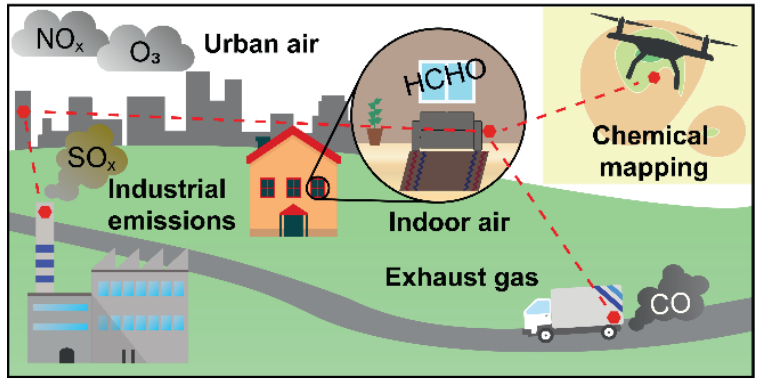

Agriculture and Food

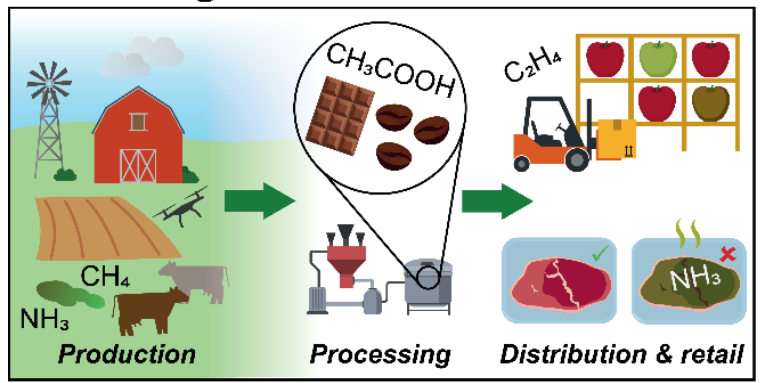

Health and Lifestyle

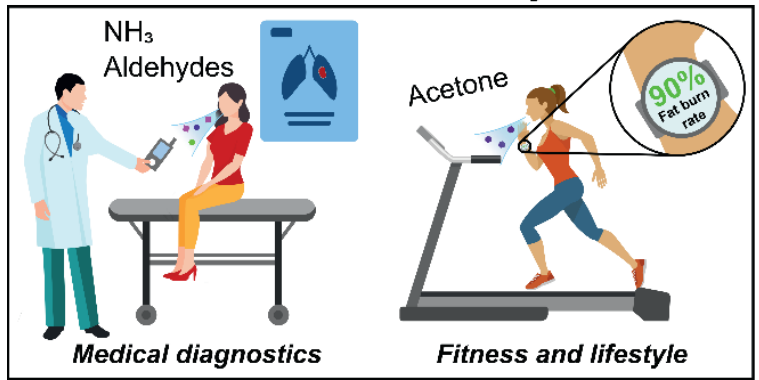

Abb. 1: Verwendung von kompakten Gassensoren zur Überwachung der Luftqualität, der Produktion von Lebensmitteln und in medizinischen Anwendungen. Reproduziert von ${ }^{[5]}$.

Besonders attraktiv für solche Anwendungen sind chemoresistive Gassensoren, basierend auf Nanopartikeln aus Halbleitern ${ }^{[6]}$. Diese sind preiswert und können auf extrem kompakten Mikrosensorsubstraten abgelagert werden, um kleinste Gaskonzentrationen (z.B. $10^{-9}$ pro Volumen an krebserregendem Formaldehyd mit Si-dotiertem $\mathrm{SnO}_{2}{ }^{[7]}$ ) zu messen ${ }^{[8]}$. Der breiten Anwendung solcher Sensoren stehen jedoch oftmals deren unzureichende Sensitivität, Selektivität und Stabilität im Weg ${ }^{[9]}$.

In diesem Übersichtsvortrag werde ich jüngste Fortschritte bei der Entwicklung hochselektiver Gassensoren präsentieren. Zuerst werde ich auf neuste Fabrikationsmethoden eingehen, welche eine verbesserte Kontrolle der Materialzusammensetzung und -morphologie ermöglichen. Dadurch können die Sensoreigenschaften maßgeschneidert für die Anwendung optimiert werden. Danach werden Filter ${ }^{[5]}$ diskutiert, welche eine systematische Optimierung der Selektivität ermöglichen. Zum Schluss wird die Integration solcher Sensoren in tragbare Messgeräte anhand ausgewählter Beispiele aufgezeigt, bevor deren Erprobung unter realen Bedingungen besprochen wird.

\section{Diskussion}

Heutzutage stehen Sensorentwicklern eine Vielzahl an Synthesemethoden zur Verfügung, welche die Zusammensetzung, Größe und Kristallphase von chemoresistiven Nanopartikeln präzise kontrollieren können. Solche Methoden werden mit speziellem Fokus auf der Flammensprühpyrolyse ${ }^{[10]}$ diskutiert, welche die Herstellung von vielversprechenden Nanopartikeln für verschiede Analyten (z.B. Si-dotiertes $\mathrm{MoO}_{3}$ für Ammoniak ${ }^{[11]}, \mathrm{WO}_{3}$ für $\mathrm{NO}_{2}{ }^{[12]}$, Ti-dotiertes $\mathrm{ZnO}$ für Isopren ${ }^{[13]}$ oder $\mathrm{Y}$-dotiertes $\mathrm{ZnO}$ für Essigsäure ${ }^{[14]}$ ) und deren Ablagerung als hochporöser Film (Abb. 2) ermöglicht. Manche dieser Sensoren können sogar bei Raumtemperatur betrieben werden (z.B. $\left.\mathrm{CuBr}{ }^{[15]}\right)$, was attraktiv für deren Integration in tragbare, batteriebetriebene Geräte ist ${ }^{[16]}$.

Danach werden Methoden der Selektivitätsverbesserung diskutiert. Zuerst werden Sensorarrays, sogenannte „elektronischen Nasen"[17], besprochen. Dabei werden mehrere solcher Sensoren (idealerweise mit orthogonalen Eigenschaften ${ }^{[18]}$ ) kombiniert ${ }^{[19]}$. Danach werden verschiedene Filterkonzepte vorgestellt, die Analyten in Gasgemischen anhand ihrer molekularen Größe (z.B. durch mikroporöse Membrane $^{[20]}$ ), Adsorptionseigenschaften ${ }^{[21-22]}$ oder Reaktivität mit ausgewählten Katalysatoren ${ }^{[23-24]}$ vorseparieren. 


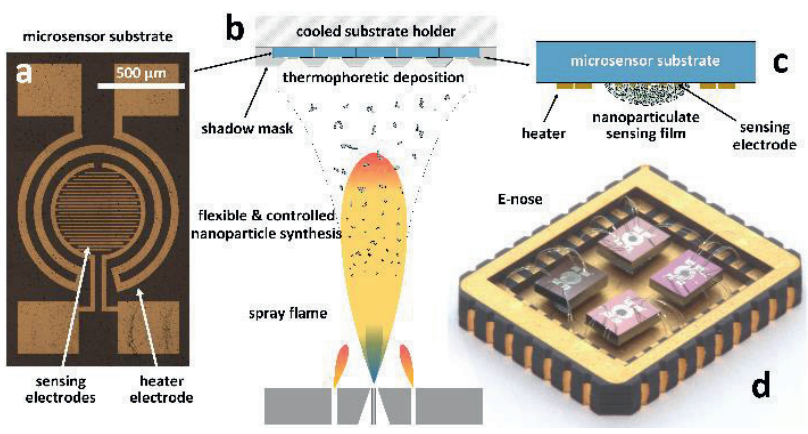

Abb. 2: (a) Bild eines Mikrosensorsubstrats. (b) Schematische Darstellung der Herstellung und Ablagerung von Nanopartikeln mit der Flammensprühpyrolyse. (c) Diese Nanopartikel werden als hochporöse Filme abgelagert, die ideal für Sensoranwendungen sind. (d) Bild mehrerer solcher Sensoren auf einem Chip. Reproduziert von ${ }^{[25]}$.

Im Folgenden werden drei konkrete Beispiele solcher chemoresistiven Sensoren und Filter präsentiert, um deren Verwendung in herausfordernden Anwendungen und den sofortigen gesellschaftlichen Nutzen aufzuzeigen. Beginnen werde ich mich einem tragbaren Methanoldetektor zur Analyse von alkoholischen Getränken, welcher die Resultate kabellos und in Echtzeit auf einem Smartphone anzeigt (Abb. 3) ${ }^{[26]}$. Der Detektor kombiniert eine SeparationskoIonne aus Tenax TA mit einem Gassensor aus Pddotiertem $\mathrm{SnO}_{2}{ }^{[27]}$, welcher dadurch Methanol selektiv zu Ethanol quantifizieren kann[28]. Dieses Messgerät kann von Schnapsbrennern ${ }^{29]}$, der Polizei oder vom Zoll zur Produktqualitätskontrolle verwendet werden, um häufig auftretende Methanolvergiftungen zu verhindern. Diese verursachten im Jahr 2019 etwa 790 Tote. Dieser Detektor funktioniert auch für Desinfektionsmitte|[30], wo Methanolverunreinigung ebenfalls ein Problem ist ${ }^{[31]}$, oder für die Atemanalyse ${ }^{[32]}$ zur Erkennung von Vergiftungen.

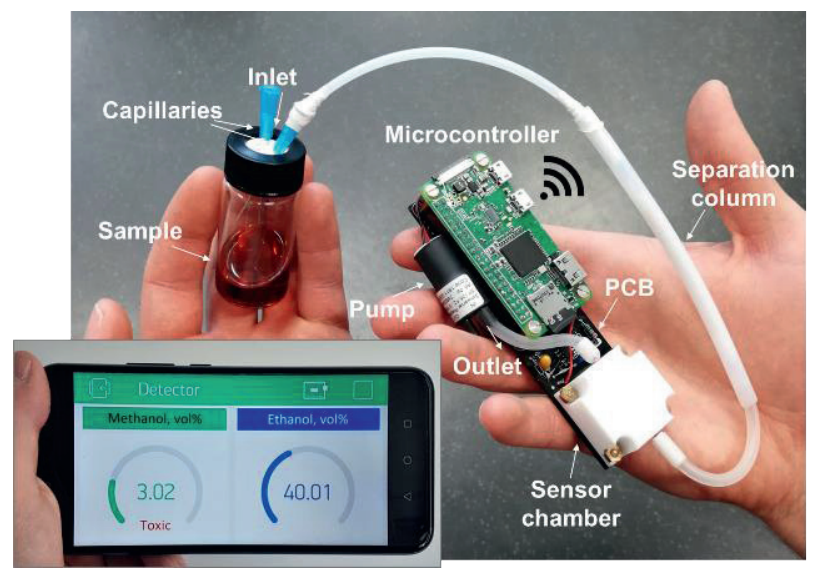

Abb. 3: Tragbarer Methanoldetektor mit kabelloser Anzeige des Messergebnisses auf einem Smartphone. Reproduziert von ${ }^{[26]}$.
Als zweites Anwendungsbeispiel werden Atemluftsensoren zur Messung von Azeton beschrieben, einem Atemmarker, der den menschlichen Fettstoffwechsel schmerzfrei anzeig $\mathrm{t}^{[33]}$. Dabei werden Sensoren aus Si-dotiertem $\mathrm{WO}_{3}$ besprochen, welche durch vorgelagerte Filter mit Pt-dotiertem $\mathrm{Al}_{2} \mathrm{O}_{3}$ das Azeton sehr selektiv in Gasgemischen (z.B. mit Ethanol, Isopren, Wasserstoff, CO) messen können. Als tragbare Atemmessgeräte ermöglichen diese die Überwachung des Fettstoffwechsels während Sport[23,34-35] oder ketogener Diät[36], um die Behandlung von Adipositas zu unterstützen. Solche Sensoren werden derzeit am Universitätsspital Zürich in klinischen Studien untersucht.

Zum Schluss wird die Verwendung solcher Sensoren zur Luftqualitätsüberwachung besprochen. Eine besondere Herausforderung ist auch hier die Messung von Luftverunreinigungen, welche selektiv bei tiefsten Konzentrationen gemessen werden müssen. Beispielsweise gilt für krebserregendes Benzol in der Europäischen Union ein Grenzwert von $80 \cdot 10^{-9}$ (pro Volumen). Dies wird durch Pd-dotierte $\mathrm{SnO}_{2}$ Sensoren mit einem vorgelagerten $\mathrm{WO}_{3}$-Filter erfüllt, welche dadurch Benzol sogar selektiv zu chemisch ähnlichem Xylol und Toluol messen können ${ }^{[37]}$.

\section{Literatur}

[1] MAYER, M.; BAEUMNER, A. J.: A Megatrend Challenging Analytical Chemistry: Biosensor and Chemosensor Concepts Ready for the Internet of Things. In: Chem. Rev. 119 (2019), S. 7996-8027.

[2] BERNA, A.: Metal oxide sensors for electronic noses and their application to food analysis. In: Sensors 10 (2010), S. 3882-3910.

[3] GÜNTNER, A. T.; ABEGG, S.; KONIGSTEIN, K.; GERBER, P. A.; SCHMIDT-TRUCKSASS, A.; PRATSINIS, S. E.: Breath Sensors for Health Monitoring. In: ACS Sens 4 (2019), S. 268-280.

[4] GÜNTNER, A. T.; PINEAU, N. J.; MOCHALSKI, P.; WIESENHOFER, H.; AGAPIOU, A.; MAYHEW, C. A.; PRATSINIS, S. E.: Sniffing Entrapped Humans with Sensor Arrays. In: Anal. Chem. 90 (2018), S. 4940-4945.

[5] VAN DEN BROEK, J.; WEBER, I. C.; GÜNTNER, A.; PRATSINIS, S.: Highly selective gas sensing enabled by filters. In: Mater Horiz 8 (2021), S. 661-684.

[6] BARSAN, N.; WEIMAR, U.: Conduction Model of Metal Oxide Gas Sensors. In: J. Electroceram. 7 (2001), S. 143167.

[7] GÜNTNER, A. T.; KOREN, V.; CHIKKADI, K.; RIGHETTONI, M.; PRATSINIS, S. E.: E-Nose Sensing of Lowppb Formaldehyde in Gas Mixtures at High Relative Humidity for Breath Screening of Lung Cancer? In: ACS Sens 1 (2016), S. 528-535.

[8] SUEMATSU, K.; HARANO, W.; OYAMA, T.; SHIN, Y.; WATANABE, K.; SHIMANOE, K.: Pulse-Driven Semiconductor Gas Sensors Toward ppt Level Toluene Detection. In: Anal. Chem. 90 (2018), S. 11219-11223.

[9] LEWIS, A.; EDWARDS, P.: Validate personal airpollution sensors. In: Nature 535 (2016), S. 29-31.

[10] MÄDLER, L.; KAMMLER, H. K.; MUELLER, R.; PRATSINIS, S. E.: Controlled synthesis of nanostructured 
particles by flame spray pyrolysis. In: J. Aerosol Sci 33 (2002), S. 369-389.

[11] GÜNTNER, A. T.; RIGHETTONI, M.; PRATSINIS, S. E.: Selective sensing of $\mathrm{NH}_{3}$ by Si-doped $\alpha-\mathrm{MoO}_{3}$ for breath analysis. In: Sens. Actuators B 223 (2016), S. 266-273.

[12] ABEGG, S.; KLEIN CERREJON, D.; GÜNTNER, A. T.; PRATSINIS, S. E.: Thickness Optimization of Highly Porous Flame-Aerosol Deposited $\mathrm{WO}_{3}$ Films for $\mathrm{NO}_{2}$ Sensing at ppb. In Nanomaterials 10 (2020), S. 1170.

[13] GÜNTNER, A. T.; PINEAU, N. J.; CHIE, D.; KRUMEICH, F.; PRATSINIS, S. E.: Selective sensing of isoprene by Ti-doped $\mathrm{ZnO}$ for breath diagnostics. In: $J$ Mater Chem B 4 (2016), S. 5358-5366.

[14] PINEAU, N. J.; KRUMEICH, F.; GÜNTNER, A. T.; PRATSINIS, S. E.: Y-doped $\mathrm{ZnO}$ films for acetic acid sensing down to ppb at high humidity. In: Sens. Actuators B 327 (2021), S. 128843.

[15] GÜNTNER, A. T.; WIED, M.; PINEAU, N. J.; PRATSINIS, S. E.: Rapid and Selective $\mathrm{NH}_{3}$ Sensing by Porous CuBr. In: Adv. Sci. 7 (2020), S. 1903390.

[16] ZHANG, J.; LIU, X.; NERI, G.; PINNA, N.: Nanostructured Materials for Room-Temperature Gas Sensors. In: Adv Mater 28 (2016), S. 795-831.

[17] PERSAUD, K.; DODD, G.: Analysis of discrimination mechanisms in the mammalian olfactory system using a model nose. In: Nature 299 (1982), S. 352-355.

[18] PINEAU, N. J.; KOMPALLA, J. F.; GÜNTNER, A. T.; PRATSINIS, S. E.: Orthogonal gas sensor arrays by chemoresistive material design. In: Microchim Acta 185 (2018), S. 563 .

[19] BROZA, Y. Y.; VISHINKIN, R.; BARASH, O.; NAKHLEH, M. K.; HAICK, H.: Synergy between nanomaterials and volatile organic compounds for non-invasive medical evaluation. In: Chem Soc Rev 47 (2018), S. 4781-4859.

[20] GÜNTNER, A. T.; ABEGG, S.; WEGNER, K.; PRATSINIS, S. E.: Zeolite membranes for highly selective formaldehyde sensors. In: Sens. Actuators B 257 (2018), S. 916923.

[21] VAN DEN BROEK, J.; CERREJON, D. K.; PRATSINIS, S. E.; GÜNTNER, A. T.: Selective formaldehyde detection at ppb in indoor air with a portable sensor. In: J. Hazard. Mater. (2020), S. 123052.

[22] VAN DEN BROEK, J.; GÜNTNER, A. T.; PRATSINIS, S. E.: Highly Selective and Rapid Breath Isoprene Sensing Enabled by Activated Alumina Filter. In: ACS Sens 3 (2018), S. 677-683.

[23] WEBER, I. C.; DERRON, N.; KÖNIGSTEIN, K.; GERBER, P. A.; GÜNTNER, A. T.; PRATSINIS, S. E.: Monitoring Lipolysis by Sensing Breath Acetone down to Partsper-Billion. In: Small Science 1 (2021), S. 2100004.

[24] GÜNTNER, A. T.; WEBER, I. C.; PRATSINIS, S. E. Catalytic Filter for Continuous and Selective Ethanol Removal Prior to Gas Sensing. In: ACS Sens 5 (2020), S. 1058-1067.

[25] GÜNTNER, A. T. Selective gas sensors from flames for breath analysis. Dissertation Nr. 23990, ETH Zürich, 2017. 10.3929/ethz-b-000181653.

[26] ABEGG, S.; MAGRO, L.; VAN DEN BROEK, J.; PRATSINIS, S. E.; GÜNTNER, A. T.: A pocket-sized device enables detection of methanol adulteration in alcoholic beverages. In: Nature Food 1 (2020), S. 351-354.

[27] PINEAU, N. J.; KELLER, S. D.; GÜNTNER, A. T.; PRATSINIS, S. E.: Palladium embedded in $\mathrm{SnO}_{2}$ enhances the sensitivity of flame-made chemoresistive gas sensors. In: Microchim Acta 187 (2020), S. 96.

[28] VAN DEN BROEK, J.; ABEGG, S.; PRATSINIS, S. E.; GÜNTNER, A. T.: Highly selective detection of methanol over ethanol by a handheld gas sensor. In: Nature Commun. 10 (2019), S. 4220

[29] PINEAU, N. J.; MAGRO, L.; VAN DEN BROEK, J.; ANDERHUB, P.; GÜNTNER, A. T.; PRATSINIS, S. E.: Spirit Distillation: Monitoring Methanol Formation with a Hand-Held Device. In: ACS Food Science \& Technology 1 (2021), S. 839844.

[30] GÜNTNER, A. T.; MAGRO, L.; VAN DEN BROEK, J.; PRATSINIS, S. E.: Detecting methanol in hand sanitizers. In: iScience 24 (2021), S. 102050

[31] U.S. FOOD AND DRUG ADMINISTRATION: Temporary policy for preparation of certain alcohol-based hand sanitizer products during the public health emergency (COVID19): Guidance for industry.

[32] VAN DEN BROEK, J.; BISCHOF, D.; DERRON, N.; ABEGG, S.; GERBER, P. A.; GÜNTNER, A. T.; PRATSINIS, S. E.: Screening Methanol Poisoning with a Portable Breath Detector. In: Anal. Chem. 93 (2021), S. 1170-1178.

[33] ANDERSON, J. C.: Measuring breath acetone for monitoring fat loss: Review. In: Obesity (Silver Spring) 23 (2015), S. 2327-34.

[34] GÜNTNER, A. T.; SIEVI, N. A.; THEODORE, S. J.; GULICH, T.; KOHLER, M.; PRATSINIS, S. E.: Noninvasive Body Fat Burn Monitoring from Exhaled Acetone with Si-doped $\mathrm{WO}_{3}$-sensing Nanoparticles. In: Anal. Chem. 89 (2017), S. 10578-10584.

[35] KÖNIGSTEIN, K.; ABEGG, S.; SCHORN, A. N.; WEBER, I. C.; DERRON, N.; KREBS, A.; GERBER, P. A.; SCHMIDT-TRUCKSÄSS, A.; GÜNTNER, A. T.: Breath acetone change during aerobic exercise is moderated by cardiorespiratory fitness. In: J. Breath Res. 15 (2021), S. 016006. [36] GÜNTNER, A. T.; KOMPALLA, J. F.; LANDIS, H.; THEODORE, S. J.; GEIDL, B.; SIEVI, N. A.; KOHLER, M.; PRATSINIS, S. E.; GERBER, P. A.: Guiding Ketogenic Diet with Breath Acetone Sensors. In: Sensors 18 (2018), S. 3655.

[37] WEBER, I. C.; RÜEDI, P.; SOT, P.; GÜNTNER, A. T.; PRATSINIS, S. E.: Handheld Device for Selective Benzene Sensing over Toluene and Xylene. In: Advanced Science accepted, DOI: 10.1002/advs.202103853. 